\title{
A high throughput screen for active human transposable elements
}

\author{
Erika M. Kvikstad ${ }^{1,2^{*}}$, Paolo Piazza ${ }^{1,3}$, Jenny C. Taylor ${ }^{1,2}$ and Gerton Lunter ${ }^{1}$
}

\begin{abstract}
Background: Transposable elements (TEs) are mobile genetic sequences that randomly propagate within their host's genome. This mobility has the potential to affect gene transcription and cause disease. However, TEs are technically challenging to identify, which complicates efforts to assess the impact of TE insertions on disease. Here we present a targeted sequencing protocol and computational pipeline to identify polymorphic and novel TE insertions using next-generation sequencing: TE-NGS. The method simultaneously targets the three subfamilies that are responsible for the majority of recent TE activity (L1HS, AluYa5/8, and AluYb8/9) thereby obviating the need for multiple experiments and reducing the amount of input material required.

Results: Here we describe the laboratory protocol and detection algorithm, and a benchmark experiment for the reference genome NA12878. We demonstrate a substantial enrichment for on-target fragments, and high sensitivity and precision to both reference and NA12878-specific insertions. We report 17 previously unreported loci for this individual which are supported by orthogonal long-read evidence, and we identify 1470 polymorphic and novel TEs in 12 additional samples that were previously undocumented in databases of insertion polymorphisms.

Conclusions: We anticipate that future applications of TE-NGS alongside exome sequencing of patients with sporadic disease will reduce the number of unresolved cases, and improve estimates of the contribution of TEs to human genetic disease.
\end{abstract}

Keywords: Transposable elements, Alu, LINE1, Polymorphism, Next generation sequencing, Bioinformatics

\section{Background}

Genome sequencing is now routinely used to identify the mutations responsible for rare genetic disease. Recent large-scale sequencing efforts of individuals with rare Mendelian disorders indicate that in approximately $20-40 \%$ of cases a causal variant can be identified (eg, [1-4]). To date, these efforts have focused mainly on small variants in exonic regions. This leaves open the possibility that a substantial fraction of the remaining causal variants are localized in non-coding regions and affect gene regulation, rather than change the proteins themselves.

One class of mutations with a potentially large effect on gene regulation are transposable element (TE) insertions. TEs are self-replicating mobile elements that

\footnotetext{
* Correspondence: erika.kvikstad@well.ox.ac.uk

${ }^{1}$ Wellcome Trust Centre for Human Genetics, Oxford, UK

${ }^{2}$ National Institute for Health Research Comprehensive Biomedical Research

Centre, Oxford, UK

Full list of author information is available at the end of the article
}

randomly insert new copies of themselves into their host's genome, with the result that in modern humans up to $60 \%$ of the genome ultimately derives from TE insertions [5, 6]. Most of these insertions were "dead on arrival" or have been inactivated over time, and today only a small collection of loci are thought to be active [7-9]. Two of these active classes of retrotransposons, LINE1 and $A l u$, copy their sequences to new locations via RNA intermediates $[10,11]$. Although the insertion rate of novel TEs is low $(\sim 1$ per 20 and 1 per 100 births for Alu and LINE1 respectively [12, 13]), together, LINE1 and Alu account for 95\% of active TE insertion in human genomes [14].

Several recent studies have highlighted the importance of this TE activity for creating population-level sequence diversity $[14,15]$. TE-mediated mutations can also cause disease by disrupting genes or modifying their expression. For instance, insertions can directly interrupt exons, causing malformed or partial protein products. In addition, because TEs contain regulatory elements 
including promoters and transcription start and stop signals, their insertion can cause nearby genes to become dysregulated or truncated (reviewed in [16]).

Because of the relative rarity of actively mobile TEs and the technical difficulty of identifying them, their contribution to human disease remains unclear. Published estimates suggest that TEs are responsible for a small fraction of human disease $(0.27 \%$, [17]), and so far, 124 individual monogenic disease-associated TE insertions have been identified [18]. However, these numbers likely represent an underestimate of the true impact of TEs, in part because they predate the clinical application of next generation sequencing (NGS) technology. Even today, most NGS assays in clinical use involve exome sequencing rather than whole-genome sequencing, and TE insertions in introns and intergenic regions with possible regulatory impact will therefore be missed.

Given their importance to population and phenotypic diversity, TE sequencing has been an active field of research including development of targeted strategies, such as amplification typing [19]. Recently, several targeted NGS technologies have been developed to detect structural variation due to TE insertions [20-28]. Strategies to target TE sequences utilize hybridization approaches in order to selectively enrich for molecules spanning a TE of interest [20,24, 27]. Alternatively, multiple degenerate primers can be used to amplify unknown flanking sequences in conjunction with a TEspecific primer $[22,28]$. Restriction enzyme digestion followed by polymerase chain reaction (PCR) has also been employed to isolate TE sequences $[21,26]$. These strategies require post-hybridization or postamplification library preparation for NGS sequencing, which substantially increases the amount of input material required (up to $10 \mu \mathrm{g}$; [26]); this is important because the amount of available genomic DNA from clinical samples is often limited. In addition, current targeted sequencing approaches typically isolate activities of either Alu [24], or LINE1 [21, 22, 26, 28], thus separate methods are required for interrogation of multiple retrotransposons in any one individual [27].

Here we present a sequencing-based whole-genome screen for polymorphic and novel TE insertions that is sensitive, specific, and cost-effective. TE-NGS simultaneously targets three major active TE subfamilies in humans: Human-specific LINE1 (L1HS), AluYa5/8, and $A l u \mathrm{Yb} 8 / 9$, together responsible for the majority (60\%) of novel TE insertions [14] (reviewed in [29]). The method creates TE-enriched libraries from genomic DNA or pre-existing genomic DNA libraries, and thus complements whole exome sequencing (WES) strategies. The procedure selectively amplifies TE sequence and the flanking regions of actively transposing TE-subfamilies. The protocol includes steps to remove amplified genomic background and sequence artefacts so that very little sequencing capacity is required. A computational pipeline then identifies the signatures of the locations of candidate novel and polymorphic TE loci.

\section{Results \\ Targeted NGS of TE insertions}

Figure 1 outlines the main workflow of the TE-NGS protocol (Methods; see Supplemental Material for detailed procedures). These steps are designed to address several challenges to targeted TE sequencing. First, the combined actively mobile elements constitute a very small fraction of the genome (0.12\%, Additional file 1: Table S1). Second, active TE of interest are similar to the large fraction of TE-derived genomic sequence [5], and in particular to the relatively abundant but inactive $A l u$ and LINE subfamilies AluSx1 and L1PA2. Third, new Alu and LINE1 elements do not insert randomly in the genome [30, 31], resulting in a subset of TE targets $(\sim 0.07 \%)$ that are clustered in close proximity and oriented in head-to-head fashion (i.e. inverted repeats; Methods; [30]), causing issues in the PCR steps of the protocol.

To overcome these challenges, we first prepare genomic libraries using standard NGS procedures followed by PCR enrichment of library fragments containing TE insertions and their flanking regions. In a single reaction, two TE-target primers are used, each designed to anneal to bases present in the Alu (AluYb8/9 and AluYa5/8), and L1HS subfamilies, respectively (Additional file 1: Table S2). These primers are complementary to the 3' ends of TE insertions, which contain motifs that distinguish the active subfamilies from closely related but inactive family members. Exponential amplification is achieved by multiplex PCR of the two TE-target primers each in conjunction with the Illumina Universal (P5) primer that will anneal to the complimentary i5 adapter present on all library molecules. Thus, many loci in the genome are amplified simultaneously without requiring degenerate primers.

Targeted sequences are expected to form at most a third of the product after this step, because in addition to exponential amplification of target elements, abundant background genome fragments lacking TE primer sites but containing the Illumina adapters (and P5 priming sites) are linearly amplified at each cycle. Additionally, target elements oriented in a head-to-head fashion will also amplify exponentially, since both ends of the fragment contain TE-primer sites. These fragments lack complementarity to the i7 Illumina sequencing primer necessary for cluster formation via bridge sequencing, and therefore do not yield usable data, but deplete the primer pool and cause issues in library normalization, affecting sequencing. 


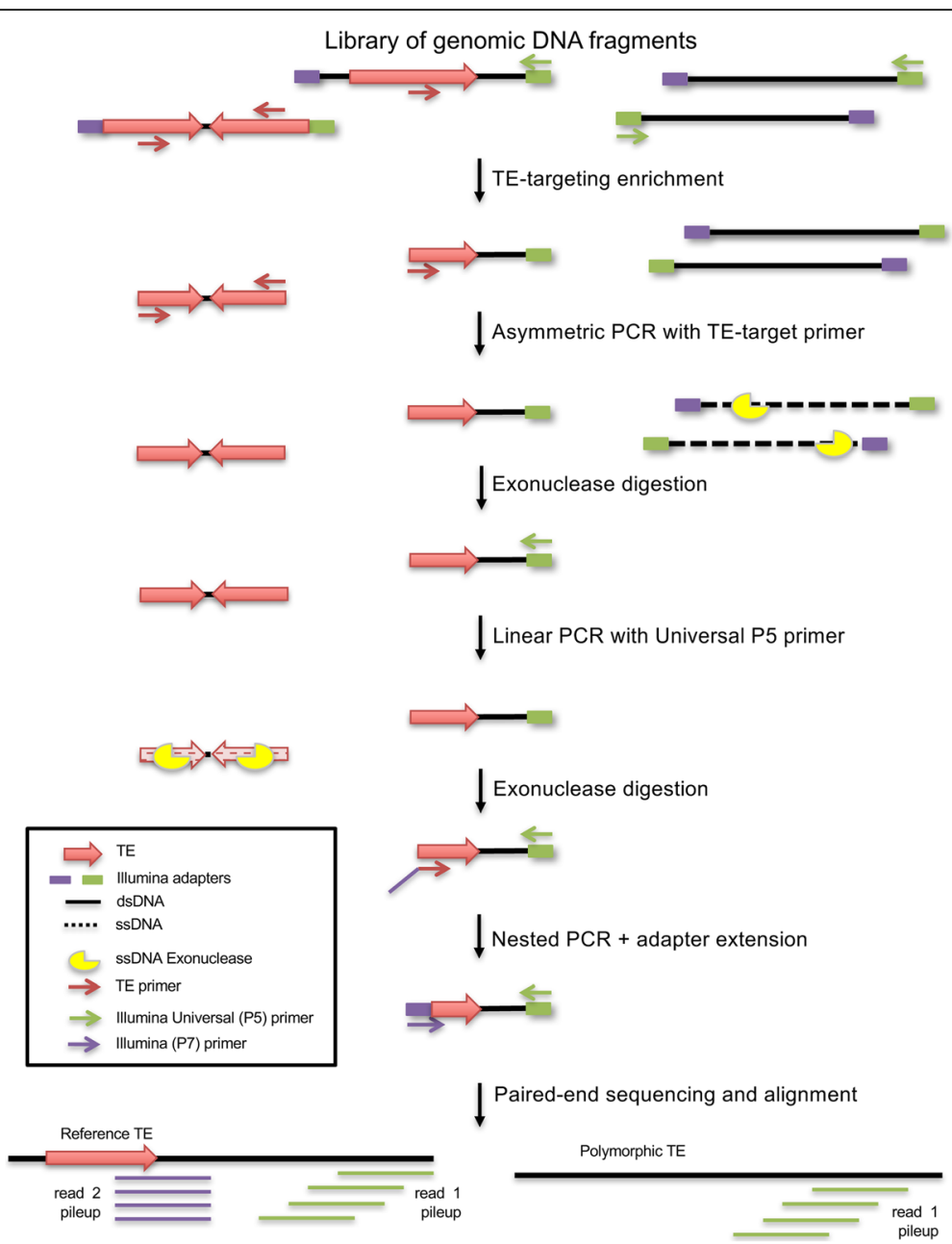

Fig. 1 TE-NGS sequencing workflow. Enrichment for genomic fragments spanning active TEs and their unique flanking sequence is achieved by several enzymatic steps as described in the main text. First, genomic DNA is sheared, and adapters for sequencing are ligated to the genomic fragments following standard library preparation protocols. Next, a small aliquot (10 ng) of library is used as template for targeted amplification with primers complementary to TE subfamily-specific sequences and to the Illumina Universal PCR (P5) primer. Remaining genomic background fragments and inverted TEs in head-to-head orientation are removed by sSDNA exonuclease digestion after linear PCR amplification with TEtarget primers or Illumina Universal primer, respectively. Last, amplification with nested primers targeting TE diagnostic bases, and containing Illumina i7 index and P7 primer sequences generates full double-stranded dual-adapter libraries containing unique indices for each sample and each TE subfamily, allowing for downstream pooling and multiplexing of many samples simultaneously. High throughput sequencing followed by alignment to the reference genome demarcates the TE insertion site by its $3^{\prime}$ end (read 2) and unique flanking sequence (read 1). TE insertions present in the reference genome can be identified by clustering of read pairs, whereas read 2 generated from polymorphic or novel TE insertions absent from the reference will map with lower quality and/or not at all; these TE can be identified by clusters of read 1 alone (see Methods; Supplemental Material for detailed procedures)

To remove these unwanted PCR products, TE-NGS libraries are processed by two rounds of asymmetric amplification and single-stranded (ss) DNA exonuclease digestion. We first perform linear amplification with TEtarget primers, leaving genomic background molecules lacking the TE largely in single-stranded form. Next, digestion by a ssDNA exonuclease selectively removes these molecules to produce a pool of dsDNA fragments containing TE targets. Similarly, molecules spanning inverted repeats are removed from the library by linear amplification with the Illumina Universal primer, so that fragments containing two TEs in head-to-head orientation, which after amplification lack adapter sequence, remain in a ssDNA configuration. After a second incubation with ssDNA exonuclease, these molecules are removed, leaving the resulting library consisting largely of fragments overlapping a single TE-target locus and its unique flanking sequence.

Finally, specificity is increased by three individual nested PCR reactions, each with a nested primer targeting a specific subfamily. At this step an Illumina index adapter is incorporated, thus producing TE-enriched 
libraries ready for multiplexing and sequencing by paired-end reads using Illumina platforms (Illumina Inc., San Diego CA; see Additional file 1: Figure S1 for representative library fragment profiles).

\section{TE identification pipeline}

TE detection from targeted NGS sequencing data is performed by a custom bioinformatics pipeline (Additional file 1: Figure S2; see Methods for details). As amplification of TEs by TE-NGS is performed with the TEnested primer in conjunction with the Universal adapter primer (P5), that is used to produce Illumina's "read 1", library fragments spanning TEs will contain Illumina read 1 generated from the unique flanking sequence, and Illumina "read 2" derived from the TE sequence (Fig. 1). These characteristics are utilized by the TE detection algorithm in making a TE call as follows.

First, reads are aligned to the reference genome. Next, read 2 are filtered by requiring the presence of TEnested primers, in addition to the presence of downstream "TE-like" sequence (see Methods for details), and both read 1 and read 2 are further filtered for mapping quality to avoid spurious calls. The remaining reads are then clustered by genomic position. TE-enriched libraries are anticipated to include TE insertions absent from the reference genome. Read 2 sequences generated from library fragments spanning such loci will contain TE sequence hallmarks (TE-nested primer, 3' portion of TE, and poly-A tail sequences) that can prevent the read from mapping reliably and/or are capable of mapping to the many near-identical sequences present in TEs located in the reference genome at alternative locations. Thus, read 1 generated from the unique TE flank is required and essential for clustering and determination TE insertion sites, whereas read 2 is not.

Last, the resulting clusters are annotated using a collection of $A l u$ and LINE insertions taken from databases of known reference TEs [32, 33] and of previously published polymorphic germline TE insertions $[14,15,23$, 25, 34]; the latter is referred to as the polymorphic TE database (polyTEdb). TE-NGS clusters are expected to fall within $600 \mathrm{bp}$ of the TE insertion site, given the starting library fragment size (Additional file 1: Figure S1). Calls produced by the above pipeline are classified as reference if the reference genome contains an insertion that matches the targeted subfamily at that position, and known non-reference if a polymorphic TE of the same class (Alu or LINE; subfamily-specific information is often not available) at that position is present in polyTEdb. Calls lacking previous evidence of a TE insertion at that position are classified as novel.

Since a minority of the genome is expected to be inaccessible due to repetitive structures, clusters close to annotated reference gaps (unassembled poly-Ns) or regions enriched for satellite repeats, such as the centromeres and sub-telomeric sequences, and the $\mathrm{Y}$ chromosome, were excluded from downstream analysis (Methods). The detailed procedures, source code and associated annotation files are available on github (https:// github.com/ekviky/TE-NGS), with documentation on a github page (https://ekviky.github.io/TE-NGS/).

\section{Performance evaluation}

We applied the TE-NGS workflow to a total of 13 human samples, resulting in 39 TE-enriched libraries (13 samples $\times 3 \mathrm{TE}$ subfamilies). Individual libraries were pooled and sequenced by $2 \times 151$-bp paired-end sequencing on a single lane of an Illumina Miseq platform (Illumina Inc., San Diego CA, USA), for a total of 25 million reads. On average, we obtained 305,609, 1,343,612, and 337,647 reads per L1HS, AluYa5/8, and AluYb8/9 library, respectively (Additional file 1: Tables S3, S4). Among the 13 human samples sequenced in this pilot experiment, we included the NA12878 individual originally sequenced by the 1000 Genomes Project and Genome in a Bottle (GiaB) consortia to benchmark the performance of the assay and detection algorithm using previously generated TE call sets $[14,15,23]$.

\section{Analysis of biochemical assay}

We first investigated the ability of the protocol to enrich for active TE families. A substantial fraction of reads from each TE-targeted library contained recognizable matches to the corresponding TE-nested primer $(0.922$, 0.949 and 0.87 for L1HS, AluYa5/8, and AluYb8/9, respectively; Additional file 1: Table S3), indicating that genomic background molecules were largely absent from the libraries.

Specificity of the assay to each targeted subfamily was assessed by comparison of observed filtered read coverage of each of the active TEs to closely related subfamilies of high sequence similarity as off-target controls. For example, L1PA2 elements that actively inserted in the common ancestor of human and chimpanzee lineages contain L1HS-nested priming sites that differ by a single nucleotide located at the ultimate 3 ' primer position, and are $\sim 3$-fold more abundant in the reference genome than L1HS is (4805 copies, Table S1; [35]). However, we observed 18.68 times more reads mapping to L1HS than L1PA2, and only a small fraction of total L1PA2 loci annotated in the reference genome were covered by 1 or more reads (14\%; Additional file 1: Table S3). Similarly, AluSx1, one of the most abundant subfamilies of AluY (109,589 loci genome-wide; Table S1) was used as control for both AluYa5/8 and AluYb8/9 experiments. We observed a substantial enrichment of reads mapping on-target vs. control (19.89 and 594.96fold for AluYa5/8 and AluYb8/9 vs. AluSx1, respectively), 
and corresponding small proportions of total genomewide AluSx1 loci in each Alu-enriched library were covered by 1 or more reads $(8.7 \%$ and $0.5 \%$, for the AluYa5/ 8 and $A l u Y b 8 / 9$ libraries respectively; Additional file 1 : Table S3).

\section{Performance of TE detection algorithm}

We next evaluated the ability of the detection algorithm to correctly discriminate true TE insertions from false predictions. To do so, we defined two sets of TE insertions to measure the overall sensitivity of the biochemical protocol, and the ability to detect de novo/ polymorphic loci in data from a well-characterized reference individual, NA12878. First, we identified a set of 624, 2739, and 1847 loci annotated as L1HS, AluYa5/8, and AluYb8/9 insertions, respectively, in the GRCh37 reference genome sequence (Reference TEs, Table 1). Since loci located in regions where NA12878 has copy number variants $(\mathrm{CNV})$ could pose alignment ambiguity or be absent altogether, we excluded reference TEs overlapping NA12878-specific CNV calls (Methods).

Second, to assess the sensitivity of the protocol to detect rare and potentially de novo loci, we identified a set of polymorphic TE calls. A number of non-reference TE calls for this individual are available through various targeted sequencing detection methods [15, 23], and computational TE discovery methods [14]. However, the limited concordance among methods leaves no clear gold standard set (e.g., Additional file 1: Figure S3, [36]). We therefore obtained 857 polymorphic Alu and 76 LINE1 mobile element insertions ("MEI") calls for NA12878 that were identified by the Phase 3 of 1000 Genomes Project [14], again excluding NA12878specific regions of CNV. This set of insertions represent the most comprehensive collection of polymorphic TE among the available call sets to date (NA12878 TEs, Table 1).

Given the limited concordance among existing call sets, and the reduced sensitivity of 1000 Genomes calls due to the low fold coverage of sequencing data produced in the project, we assessed specificity of the protocol using the union of the polymorphic NA12878 insertions available. Thus, predictions of our pipeline for which no evidence of a TE insertion at that position exists among all available call sets were considered false positives (FP).

True positives (TP) were defined as predicted calls having a corresponding TE in the set of Reference TEs within $600 \mathrm{bp}$ from the annotated 3' end of the repeat insertion, and that matches the subfamily annotation. Because only a small number of NA12878 MEI calls in the 1000 Genomes have complete subfamily annotation (163 AluYa5/8 and 61 AluYb8/9), we only required a match to the repeat class (Alu or LINE1) to consider NA12878 true positives.

False negatives (FN) were defined as Reference TEs and NA12878 TEs annotated as L1HS, AluYa5/8, or AluYb8/9 for which no corresponding call in our dataset exists within $600 \mathrm{bp}$ of the annotated $3^{\prime}$ end of the repeat insertion. We restricted 1000 Genomes Phase 3 MEI calls to only those with the AluYb8/9 and AluYa5/8 subfamily annotation for determining NA12878 Alu false negatives. MEI calls lacking subfamily annotation that were not observed among our calls were not counted towards FNs, since our method is designed to be specific to active AluYb8/9 and AluYa5/8 and not older, dormant elements (e.g., AluS, AluJ). However, since none of the LINE1 MEI calls have any subfamily designations, in order to be able to compute a NA12878-specific FN rate we conservatively assumed that all 76 of the LINE1 MEI calls were in fact L1HS insertions.

To characterize the ability of TE-NGS to predict true insertions, we evaluated the recall (sensitivity), computed as $\mathrm{TP} /(\mathrm{TP}+\mathrm{FN})$, for Reference and NA12878 TE sets. Precision, computed as TP/(TP + FP), was used to quantify the ability of the assay to reject false insertion predictions. As illustrated in Fig. 2, TE-NGS achieves high rates of recall when considering all loci that are supported by at least one read $(0.97,0.92,0.94$ reference L1HS, AluYa5/8, and AluYb8/9, respectively). For example, we observed 33/35 full length MEI LINE1 events annotated in NA12878. However, at this 1-read

Table 1 TE loci observed in NA12878 NGS libraries

\begin{tabular}{lllllll}
\hline TE library & Reference TP & Reference FN & NA12878 TP & NA12878 FN & FPe $^{\text {d }}$ & Validated Novel $^{f}$ \\
\hline L1HS & $589(84642)$ & 35 & $54(1493)$ & 22 & $19(74)$ & $10(38)$ \\
AluYa5/8 & $2335(51529)$ & 404 & $143(874)$ & 91 & $9(44)$ & $6(32)$ \\
AluYb8/9 & $1664(61099)$ & 183 & $119(953)$ & 29 & $3(12)$ & $1(4)$ \\
\hline
\end{tabular}

${ }^{2}$ Reference TP, observed TE insertions (reads) in the reference truth set with a TE cluster within 600 bp window of 3 ' terminal position and match to predicted TE subfamily. Clusters contain filtered reads with a minimum 2 or more Illumina read 1 derived from the unique flanking sequence. See text for details

${ }^{\mathrm{b}}$ Reference $\mathrm{FN}$, false negatives computed as reference TE subfamily members lacking cluster within 600 bp window of TE 3 ' terminal position

'NA12878 TP, observed 1000 Genomes Phase 3 MEl calls in NA12878 having an identified TE cluster within 600 bp window of 3' terminal position and matching predicted TE class (Alu, LINE1)

${ }^{\mathrm{d}} \mathrm{NA} 12878 \mathrm{FN}, \mathrm{MEI}$ calls with TE subfamily classification lacking an observed cluster within $600 \mathrm{bp}$ window of TE 3 ' terminal position

eFP, false positive clusters lacking previous evidence of TE insertion within $600 \mathrm{bp}$ window of cluster position before validation with GiaB and ONT long-read data

fValidated Novel, FP clusters supported by evidence from GiaB and ONT long-read data 


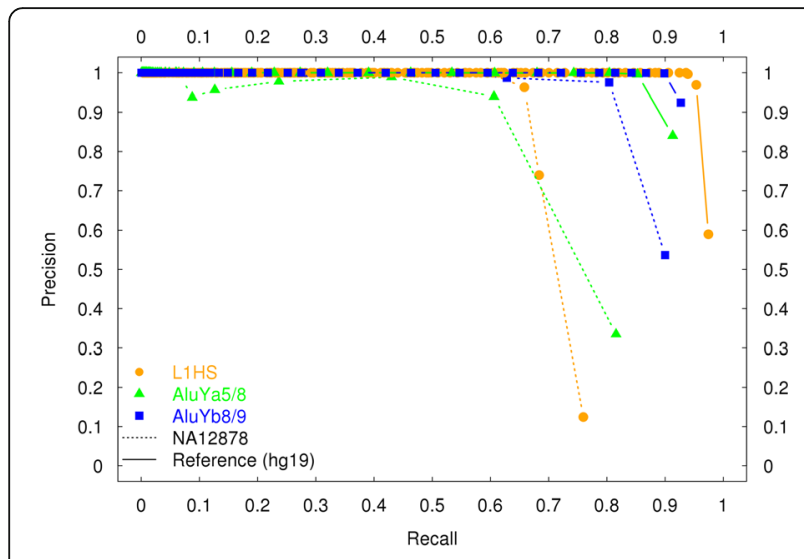

Fig. 2 Precision and recall as a function of cluster read depth. Performance of TE insertion detection was assessed computing precision and recall separately for reference and polymorphic TEs present in NA12878. Reference (NA12878) true positives (TP) and false negatives (FN) were determined by comparison of clusters to reference (hg19) RepeatMasker annotations (NA12878-specific 1000 Genomes Phase $3 \mathrm{MEl}$ calls), respectively. False positives (FP) were defined conservatively as TE candidate clusters failing to intersect any previously identified NA12878 non-reference events (see main text; Methods for details)

threshold many spurious calls are made that lack previous evidence of an insertion. We therefore explored precision and recall as a function of cluster read depth (Fig. 2) to determine an effective threshold for defining a TE insertion call. A reasonable balance between sensitivity and specificity was achieved when we required a minimum of 2 reads per cluster derived from the unique flanking sequence 3 ' to the insertion site. At this threshold, we observed $0.95,0.85$ and 0.90 Reference (0.71, 0.61, 0.80 NA12878-specific) TE insertions, with precision rates of $0.97,0.99$, and 0.99 for reference calls $(0.74$, 0.94, 0.98 for NA12878-specific calls) for L1HS, AluYa5/ 8, and AluYb8/9, respectively (Fig. 2, Table 1; Methods). A complete list of all annotated TE calls identified in NA12878 is available as a flat file on github (https:// github.com/ekviky/TE-NGS).

\section{Validation of NA12878 TE calls with long read data}

We considered the possibility that a fraction of TE-NGS predictions in NA12878 that were classified as false positive could be caused by annotations missing from the 1000 Genomes Phase 3 MEI call set and the other call sets we considered. To address this possibility, we examined long-read sequence data for NA12878 for evidence of TE insertions at calls classified as FP. Long reads averaging $>10,000 \mathrm{bp}$ [37] are capable of spanning the entirety of a TE insertion in the case of Alus ( $300 \mathrm{bp})$, and much if not all of LINE1 that are typically truncated upon insertion ( 500 bp and longer; [38]).

First, we obtained sequence data of NA12878 PacBio reads generated by the GiaB consortium [37, 39]. We interrogated these data for sequence signatures consistent with TE insertions. PacBio reads spanning FP calls and containing exact matches to TE-like sequences validated a total of $27 / 31(0.87)$ FP as likely true insertions. By subfamily, 18/19, 7/9, and 2/3 L1HS, AluYa5/8, and AluYb8/9 FP calls, respectively, are represented by at least one read with TE-like sequence, with mean coverage depth of 31.26 (median 9) TE-like reads per locus.

Second, FP were manually inspected to detect evidence consistent with TE insertions using an orthogonal long read data set for NA12878 generated by Oxford Nanopore Technology (ONT, [40]). Visual inspection of these data confirmed that 20/31 (0.65) of FP calls are spanned by ONT reads consistent with TE insertion (Additional file 1: Figure S4, Methods).

Together, we find that at least one of the long-read data sets provided independent evidence supporting the presence of a TE insertion at 30/31 loci that were considered FPs in the initial analysis; 17/31 loci (10/19 AluYa5/8, 6/9 AluYb8/9 and 1/3 L1HS) were supported by evidence from both GiaB and ONT reads. This resulted in increased precision rates for both reference and polymorphic TE sets; using the more conservative estimate we find a precision of 0.985 (589/598), 0.999 (2335/2338) and 0.999 (1664/1666) for L1HS, AluYa5/8, AluYb8/9 reference calls respectively, and $0.86(54 / 63)$, 0.98 (143/146), and $0.98(119 / 121)$ for NA12878-MEI calls of the same types (Table 1 ). The 14 remaining FP calls represent a small fraction, $0.29 \%$ (14/4904), of total polymorphic TE predictions produced by this method.

\section{Application of TE-NGS to additional samples}

Twelve additional clinical samples were sequenced by the TE-targeting method along with NA12878. These samples correspond to individuals with previously generated WES data and for whom no causal variants were identified. Additional file 1: Table S4 summarizes the TE calls produced by application of TE-NGS. One individual (sample 8) failed all three targeting experiments and was excluded from further analysis. On average, in these samples we observe a fraction of $0.90,0.87$, and 0.88 of the expected reference L1HS, AluYa5/8, and AluYb8/9, respectively, similar to the observed rates of recall for NA12878 individual (see above). In conjunction with the TE hallmarks utilized by TE-NGS calling algorithm, 0.99 (943744/948311), 0.95 (9573734/10096770), and 0.99 (904347/913524) of reads spanning all reported L1HS, AluYa5/8, and AluYb8/9 calls, respectively - including reference, known non-reference, and novel - can be corroborated by identification of poly-A tails, an additional feature of retrotransposition.

We detected a total of 1470 novel TE insertions, 1438 of which are unique. By subfamily, this breaks down as an average of $42.5,80$, and 14 novel insertions of L1HS, 
AluYa5/8, and AluYb8/9 per individual (Additional file 1: Table S4). Two percent (32/1470) of the novel events were observed in more than one individual, including two previously unreported insertions of L1HS that were observed in all 11 samples (chr2: 132,998,699132,998,918; chr16: 61,079,102-61,079,356; both build GRCh37). The distribution of all identified novel insertions largely follows the genome-wide expectation, with no discernable chromosome bias nor enrichment near genes (data not shown).

\section{Estimation of performance using trio data}

Given the low estimated insertion rates $(\sim 1$ per 20 births for Alu; see above), we expect that the vast majority of novel TE insertions are rare polymorphic events inherited from either parent, and/or false positives, rather than de novo insertions. For two sets of trios included in this study, inheritance patterns were ascertained at TE loci observed in each proband, allowing us to estimate false positive and false negative calls. We evaluated sensitivity for the parental truth set (defined as clusters present in both parents), and novel TE insertions unique to the proband (absent in both parents) were considered as false positives calls for calculation of precision and recall (see Methods for details).

Of the 5692 clusters present in both parents of Trio A, we observed 5384 (95\%) in the proband (sample 1); broken down by TE subfamily, the proposed method's recall for this individual was 682/721 (0.95), 2972/3110 (0.96), and 1730/1861 (0.93) for L1HS, AluYa5/8, and AluYb8/9, respectively. For example, Additional file 1: Figure S5 illustrates a novel L1HS observed in all members of Trio A and thus inferred to be inherited in the proband. Of the total novel calls detected in this proband, 7/11 L1HS and 22/29 AluYa5/8 were absent from both parents and therefore classified as false positives, while every novel AluYb8/9 was inherited, corresponding to high rates of precision $(>=0.99)$ for each assay $(682 /$ 689, 2972/2994, 1730/1730 for L1HS, AluYa5/8, and AluYb8/9, respectively; Additional file 1: Table S4; Methods).

The Trio B parental truth set contained 6156 clusters, 5756 (94\%) of which were observed in the proband (sample 4; Additional file 1: Table S4). In this particular individual, we observed 690/744 (0.93), 3258/3450 (0.94), and 1808/1962 (0.92) rates of recall for L1HS, AluYa5/8, and AluYb8/9, respectively. And similarly high rates of precision 0.89 (690/773), 0.94 (3258/3481), 0.98 (1808/1841) were obtained for L1HS, AluYa5/8, and AluYb8/9, respectively.

\section{Discussion}

We have developed a method for the identification of novel TE elements in human genomes, consisting of a molecular genomics protocol and bioinformatics pipeline (Fig. 1, Additional file 1: Figure S2). TE-NGS uses targeted NGS sequencing combined with genomic positional evidence to identify TE insertions, and novel loci are discriminated by further annotation with public databases. The approach here simultaneously targets the three elements responsible for the majority of active retrotransposition in the human lineage (AluYa5/8, AluYb8/9, L1HS). Using the well-characterized human sample NA12878 we show that the approach achieves high sensitivity and specificity (Fig. 2).

To determine the genomic locations of novel TE insertions, an assay must amplify not only the TE itself but the unique contextual sequence of each element. We achieved this by taking advantage of the Universal adapter primer present in Illumina genome libraries, using PCR to selectively amplify only the subpopulation of genomic molecules that span the junction of a TE insertion (Fig. 1).

The presence of many near-identical repeat inserts, and the large amount of genomic background sequences over TE-containing genomic molecules present challenges to generating on-target and usable data. In addition, molecules spanning inverted TE pairs in headto-head orientation will propagate through the protocol, and are capable of hybridizing to Illumina flowcells, but as they consist of TE primer sites at both ends they contain only one of the two adaptors required for cluster formation, producing molecules ultimately incompatible with dual-adapter NGS sequencing and reducing the yield of the protocol. We used two incubations with an ssDNA exonuclease to systematically degrade these unwanted PCR products from the libraries (Fig. 1), allowing us to decrease genomic background DNA and remove molecules spanning inverted repeats. Introducing exonuclease digestion steps resulted in high proportions ( $87 \%$ or more; Additional file 1: Table S3) of usable read data generated for each element.

The proposed protocol significantly improves upon on the total cost and sample usage compared to previous approaches. Current approaches require up to tens of millions of reads per sample e.g., [23, 26, 27], offsetting the potential for deep multiplexing to decrease the burden of sequencing costs. By contrast, the protocol described here allowed us to sequence 39 libraries (13 samples $\times 3$ elements) on a single Miseq lane. Given the levels of sensitivity attained (Fig. 2) and the complexity of the resulting libraries (averaging 143, 22 and 36 reads per L1HS, AluYa5/8, and AluYb8/9 locus, respectively; Table 1), we estimate that as many as 400 individuals could be multiplexed on a single lane of Illumina HiSeq 2500 (assuming a minimum of $200 \mathrm{M}$ reads, and $\sim 500,000$ reads/sample). Moreover, only $10 \mathrm{ng}$ of genomic library is required as input material; many library preparation kits perform well 
with as little as 5 ng of genomic DNA (e.g., New England Biolabs, Ipswich, Massachusetts).

Despite these gains, several areas remain for further improvement. The utility of the protocol would increase if the relatively high FN rate of $\sim 5 \%$ could be decreased. In part, $\mathrm{FN}$ are driven by sequence characteristics of the TE flanking sequences which influence the ability of the relatively short reads to map uniquely. Some FNs will be due to reference TEs that overlap CNVs in the particular individual. Unfortunately, Phase3 calls for TE deletions with respect to the reference genome are not readily available, such that a complete set of reference insertions known to be absent from NA12878 is not available at this time. Another cause of FNs is the variable coverage between individual TE loci, due to differential efficiencies in the various PCR steps in the protocol, which contribute to an underrepresentation of reads at some loci that could be mitigated by increasing the overall depth of sequencing.

We find that the observed detection rate for polymorphic NA12878 TEs is lower than that for Reference TEs. This could be due in part to the incomplete nature of Phase3 MEI annotations. For example, Phase3 MEI calls are annotated as LINE1 events, while our assay is designed to target specifically the active (Ta-0 and Ta subset) of L1HS subfamilies. It is possible that a proportion of these loci correspond to L1PA2 or other closely related, yet inactive subfamilies that will not be targeted by TE-NGS (Additional file 1: Table S3). Indeed, of the 35 full length, i.e. presumed active, LINE1 events in Phase 3 calls, we observe all but 2 (94\%), consistent with recent assays targeting the "hot" and active subset of LINE1 events [28]. Furthermore, inspection of long-read data produced by both Pac Bio and ONT confirmed substantial proportions $(18 / 19,7 / 9,2 / 3)$ of FP L1HS, AluYa5/8 and AluYb8/9 calls as likely true insertion events absent from the 1000 Genomes Phase3 call set.

The results of the PacBio and ONT long-read analysis also suggest that TE-NGS achieves high specificity for targeted TE subfamilies. Although the precision values presented here are conservatively calculated using calls validated by both PacBio and ONT reads, it is unlikely that positive evidence of a TE in a long and uniquely mappable ONT or PacBio read would occur by chance. Discrepancies between PacBio and ONT data could be explained by variable coverage in each data set, as well as a minority of unique insertions in the particular NA12878 cell lines utilized by each experiment.

The capacity to detect all TEs in an individual will require measures to sequence and align reliably in difficult genomic contexts. Although this protocol was developed for use with Illumina sequencing platforms, in theory, any existing NGS technology can be used insofar as the appropriate adaptor sequences and sequencing primers are publicly available for use in primer design. This has not been tested directly at this time, yet emerging long-read technologies may allow future adaptations of the protocol to sequence the TE and both its breakpoints in entirety. Long reads could also lead to enhanced sensitivities in difficult genomic regions (e.g., nested insertions, inverted repeats).

Recent advances in NGS technology have decreased the costs for whole genome sequencing (WGS). In theory, short-read WGS should allow most TE insertions to be detected irrespective of TE class/family. Several computational tools (reviewed in [41]) have been developed to detect structural variation in WGS data due to TE insertion. However, even with today's sequencing platforms WGS remains expensive. It should be noted that even with WGS data, existing computational tools show low concordance, even at high read coverage [36].

\section{Conclusions}

Remaining challenges notwithstanding, the comprehensive nature of TE-NGS, its high sensitivity and precision, and its cost-effectiveness due to the high fraction of ontarget data, opens the possibility of adding effective TE screening to existing sequencing projects. This is particularly true for whole-exome sequencing projects, where TE-NGS can reuse part of the library preparation protocol to detect potential clinically relevant mutations that are not targeted by whole-exome methods. Importantly, the method described here provides an affordable assay for detecting novel TE insertions, a significant source of structural variation in human genomes that is not currently investigated in detail and may be more relevant in clinical settings than is currently appreciated.

\section{Methods \\ Samples}

We obtained 12 NGS genomic libraries previously sequenced by the Oxford Genomics Centre from individuals recruited as part of the Oxford Biomedical Research Centre's Clinical Exomes project. The genomic library for individual NA12878 was kindly provided by Oxford Genomics Centre.

\section{TE primer design}

To selectively enrich NGS libraries for TE-containing fragments, PCR primers were designed to amplify actively transposing members of Alu (Yb8/9 and Ya5/8) and L1HS (Ta-1 or 0 ) subfamilies (Additional file 1: Table S2). Primers were designed using Primer3 software with the following characteristics: (i) targeting diagnostic nucleotides specific to each subfamily; (ii) positioned in proximity to $3^{\prime}$ tail of the consensus sequence to optimize coverage of flanking sequence; and (iii) containing low complementarity to facilitate multiplexing in 
single PCR reactions [42]. Each element was targeted by two primers. First, two TE-target primers were designed to amplify the subfamilies of interest, one primer each for Alu and LINE1. Second, three TE-nested primers complementary to subfamily-specific bases located further downstream ( $\left.3^{\prime}\right)$ from the targeting primer. The L1HS-target primer, L1HsTailSp2, was previously published ([23], Additional file 1: Table S2).

Primer subfamily specificity was measured by determining the number and locations of primer matches in the reference genome (hg19) allowing at most 1 mismatch at positions excluding the 3 ' ultimate nucleotide, and intersecting matches with RepeatMasker annotations [32] obtained from UCSC Table Browser [43].

TEs located in head-to-head orientation, i.e. inverted repeats capable of exponential amplification, were identified by defining genomic positions of all possible combinations of TE-target primer and a primer's reverse complement (likewise TE-nested primer and reverse complement). Using the same defined primer match parameters (see above) and limiting primer distances to within a maximum of 1-kb (estimated maximum library fragment size), we identified 352 loci as inverted repeats.

\section{TE-NGS library preparation}

\section{Preparation of genomic libraries}

First, genomic molecules were sheared to an average library insert size of $\sim 300$ bp using Covaris following manufacturers specifications (Covaris, Woburn, Massachussets). Library construction consisting of end repair, adapter ligation and PCR extension was performed using NEBNext Ultra DNA Library Kit for Illumina (New England Biolabs, Ipswich, Massachusetts; cat. E7370) to generate libraries with full-length adapters including index 1 (i7) for Illumina pairedend sequencing.

\section{TE-target amplification}

TE-containing fragments were exponentially amplified from genomic library using the two TE-targeting primers in conjunction with the Illumina Universal PCR primer (P5) in a multiplex reaction (Additional file 1: Table S2). The PCR reaction was performed in a $50 \mu \mathrm{L}$ volume containing NEB Q5 High Fidelity $2 \times$ PCR mastermix (New England Biolabs; cat. M0541 L), each TE primer at concentration of $0.5 \mu \mathrm{M}, 1 \mu \mathrm{M}$ of Illumina Universal P5 PCR primer, $2 \mathrm{mM}$ of magnesium, and $\sim 10 \mathrm{ng}$ of library DNA. PCR cycling conditions were as follows: initial denaturation at $98{ }^{\circ} \mathrm{C}$ for $2 \mathrm{~min} ; 10$ cycles of (denaturation at $98{ }^{\circ} \mathrm{C}$ for $10 \mathrm{~s}$, annealing at $67{ }^{\circ} \mathrm{C}$ for $30 \mathrm{~s}$, extension at $72{ }^{\circ} \mathrm{C}$ for $30 \mathrm{~s}$ ); and final extension at $72{ }^{\circ} \mathrm{C}$ for $5 \mathrm{~min}$.

\section{Post-PCR DNA purification}

DNA purification and library size selection was performed by solid phase reversible immobilization (SPRI) using Agencourt AMPure XP magnetic beads (Beckman Coulter, Brea, California; cat. A63882). A ratio of $1.8 \times$ beads to reaction volume was used to selectively retain fragments $\sim 100 \mathrm{bp}$ and larger, and to ensure removal of Illumina P5 primers from the reaction solution.

\section{TE-target asymmetric amplification \& digestion of background ssDNA fragments}

To asymmetrically amplify fragments containing TEtargeting primer sites, a PCR reaction was performed with NEB Q5 High Fidelity $2 \times$ PCR mastermix, both TE-target primers at $0.5 \mu \mathrm{M}$ each, $2 \mathrm{mM}$ magnesium, and clean eluted PCR reaction from previous step. Cycling parameters were denaturation at $98{ }^{\circ} \mathrm{C}$ for $2 \mathrm{~min}$, followed by 2 cycles of (denaturation at $98{ }^{\circ} \mathrm{C}$ for $10 \mathrm{~s}$, annealing at $67{ }^{\circ} \mathrm{C}$ for $30 \mathrm{~s}$, and extension at $72{ }^{\circ} \mathrm{C}$ for $30 \mathrm{~s})$.

Double-stranded DNA (dsDNA) products at this step include linear amplification from TE targets, and exponential amplification of TE targets oriented in head-to-head fashion. Background genomic DNA molecules lacking TEtarget priming sites remain largely denatured (due to their high complexity), in single-stranded DNA (ssDNA) form. To remove the unwanted ssDNA fragments from the library after asymmetric amplification, libraries were incubated with Recff exonuclease (New England Biolabs, cat. M0264 L) following manufacturer's specifications.

\section{Post-digest DNA purification}

Libraries following exonuclease digestions were purified by SPRI using AMPure XP magnetic beads in a $1.8 \times$ ratio of beads to reaction volume, to selectively retain fragments $\sim 100 \mathrm{bp}$ and larger and to remove TE-target primers from the reaction solution.

\section{Illumina adapter (P5) asymmetric amplification \& digestion of head-to-head ssDNA fragments}

To asymmetrically amplify fragments containing the Illumina universal adaptor sequence, a linear PCR reaction was performed with Illumina P5 PCR primer at $0.5 \mu \mathrm{M}, \mathrm{NEB}$ Q5 High Fidelity $2 \times$ PCR mastermix, and clean eluted PCR reaction from previous step. Cycling parameters were denaturation at $98{ }^{\circ} \mathrm{C}$ for $2 \mathrm{~min}$, followed by 2 cycles of (denaturation at $98{ }^{\circ} \mathrm{C}$ for $10 \mathrm{~s}$, annealing at $63{ }^{\circ} \mathrm{C} 30 \mathrm{~s}$, and extension at $72{ }^{\circ} \mathrm{C} 30 \mathrm{~s}$ ).

At this step, dsDNA products will result from linear amplification of TE targets. Molecules containing TEs in head-to-head orientation lack Illumina universal adapters, and remain largely denatured in ssDNA form, for subsequent removal by exonuclease digestion. 
Digestion was performed by incubation with RecJf exonuclease as above.

\section{TE-nested amplification}

To further increase specificity, three individual PCR reactions were performed each targeting a specific subfamily. TE-nested primers were designed for further TE specificity, and contain nucleotides to partially reintroduce the Illumina index i7 adapter that contains a unique 6-mer index per TE, per sample (Additional file 1: Table S2). Each PCR reaction was performed in a separate $50 \mu \mathrm{L}$ volume containing a $4 \mu \mathrm{l}$ aliquot of the postdigest library from previous step, NEB Q5 High Fidelity $2 \times$ PCR mastermix, one TE-nested primer at concentration of $0.5 \mu \mathrm{M}$, and Illumina P5 Universal adapter primer at $0.5 \mu \mathrm{M}$. PCR cycling conditions were as follows: initial denaturation at $98 \mathrm{C}$ for $2 \mathrm{~min}$; 15 cycles of (denaturation at $98{ }^{\circ} \mathrm{C}$ for $10 \mathrm{~s}$, annealing at Ta_primer for $30 \mathrm{~s}$, extension at $72{ }^{\circ} \mathrm{C}$ for $30 \mathrm{~s}$ ); and final extension at $72{ }^{\circ} \mathrm{C}$ for $5 \mathrm{~min}$; where Ta_primer corresponds to $\left(68{ }^{\circ} \mathrm{C}\right.$ L1HS-nested, $68{ }^{\circ} \mathrm{C}$ AluYa58-nested, and $64{ }^{\circ} \mathrm{C}$ AluYb89-nested primer, respectively; see Supplemental Material for detailed procedures; Additional file 1: Table S1).

\section{Post-PCR DNA purification}

Libraries following nested amplification were purified by SPRI using AMPure XP magnetic beads in a $1.8 \times$ ratio of beads to reaction volume, to selectively retain fragments $\sim 100$ bp and larger.

\section{Adapter extension}

Enrichment for molecules containing full-length doublestranded DNA adapter libraries was achieved by PCR adapter extension. A $50 \mu \mathrm{L}$ PCR reaction was performed with Illumina P5 Universal PCR primer and P7 index primers at $0.5 \mu \mathrm{M}$ each, NEB Q5 High Fidelity $2 \times$ PCR mastermix, and clean eluted PCR reaction from previous step. Cycling parameters were denaturation at $98{ }^{\circ} \mathrm{C}$ for 2 min, 5 cycles of (denaturation at $98{ }^{\circ} \mathrm{C}$ for $10 \mathrm{~s}$, annealing at $65{ }^{\circ} \mathrm{C}$ for $30 \mathrm{~s}$, and extension at $72{ }^{\circ} \mathrm{C}$ for $30 \mathrm{~s}$ ), and final extension at $65^{\circ} \mathrm{C}$ for $5 \mathrm{~min}$.

\section{Library multiplexing and sequencing}

The resulting TE-enriched libraries were purified, quantified then pooled according to relative molarities. Full length adapter libraries were purified by SPRI using AMPure XP magnetic beads in a $1 \times$ ratio of beads to reaction volume, to selectively retain fragments $\sim 150 \mathrm{bp}$ and larger. The molarity of each library was computed by obtaining the concentration using Qubit (Life Technologies, Carlsbad, California) and size distribution on Agilent Tapestation High Sensivity D1000 Screen Tape (Agilent Technologies, Santa Clara, California; cat. 5067-5584).
Additional file 1: Figure S1 demonstrates the fragment distribution of a typical library, that is free of short ( $<100 \mathrm{bp}$ ) fragments and displays a peak at $\sim 200 \mathrm{bp}$, or approximately half the size of the starting library.

TE-enriched libraries were subsequently multiplexed according to relative molarities (see Supplemental Material). The final library pool was diluted to $10 \mathrm{nM}$ and sequenced by $2 \times 151$-bp paired-end reads using Illumina MiSeq (Illumina Inc., San Diego CA).

\section{Identification of TE loci from targeted sequencing Alignment}

Sequence data generated by TE-enrichment were demultiplexed and aligned to the human reference genome (hg19) using the Burrows-Wheeler Aligner [44] followed by refinement of BWA alignments with Stampy [45], sorting of BAM alignments using Samtools sort [46], removal of PCR duplicates using the module MarkDuplicates in Picard [47] and BAM indexing using Samtools index.

\section{Post-alignment preprocessing of reads}

TE loci in the targeted sequencing data were identified by a bioinformatic pipeline that incorporates signatures of TE loci such as (i) primer matches (ii) matches to "TE-like" sequence (iii) read orientation (Additional file 1: Figure S2). Amplification of TEs by TE-NGS is performed with the TE-nested primer in conjunction with the Universal adapter primer (P5), that is used to produce Illumina's "read 1". Therefore, Illumina read 1 (derived from Illumina i5/P5 primer) will be generated from the unique flanking sequence, while Ilumina "read 2" (produced from i7/P7 primer) will contain primer, 3' portion of TE, and poly-A tail sequences (Fig. 1). As such, different requirements were placed on the two reads generated from the targeted paired-end sequencing as follows.

Reads derived from the mobilome were distinguished from genomic background by requiring exact matches to the full-length TE-nested primer sequences at the start of read 2. This strict filtering minimized the potential for false positives calls. Read pairs with read 2 lacking any discernable match to the primer (defined as exact matches to first 7/10 nucleotides of primer) correspond to genomic background and were removed from further analysis.

Because sites in the genome with complementarity to TE-nested primers could amplify exponentially and produce spurious calls, we investigated reads in detail for TE hallmarks, in particular the 3' portion of TE consensus sequence (referred to in text as "TE-like" sequence). So-called TE-like sequences were defined as sequence downstream from the TE-nested primer, i.e. (3') relative to the TE sequence, and excluding the poly-A tail. TE- 
like sequence was determined for each TE subfamily by analysis of consensus sequences obtained from Repbase [33]. Multiple alignments were produced separately for all Alu and all LINE1 subfamily sequences using MUSCLE [48]. Based on the position of the TE-nested primer in the consensus sequence, the length of TE-like sequence varies for each subfamily $(8,37$, and 29 nucleotides for L1HS, AluYa5/8 and AluYb8/9, respectively).

We examined the distribution of mismatches to TE consensus sequences in filtered read 2 that contain exact matches to the TE-nested primers. Additional file 1: Figure S6 illustrates the density of mismatches to the consensus sequence for each position, and the frequency of mismatches in the $3^{\prime}$ portion of AluYb8/9 clusters as a representative target. We observe that the proportion of sequences with mismatches differs significantly for reads overlapping Reference TP (0.086) and NA12878 TP (0) vs. putative FP loci (0.72; both $p<2 \times 10^{-16}$; Chisquare test, 2 degrees of freedom). Thus, TE-like sequence was implemented as a classifier by the TE-NGS calling algorithm, and we defined mismatch thresholds for discrimination of TP from FP loci. Read 2 reads with a maximum number of allowed mismatches to the TElike sequence $(3,10$, and 10 for L1HS, AluYa5/8 and AluYb8/9, respectively) were considered TE-derived and their read pairs retained (Additional file 1: Figure S6).

TE-enriched libraries are anticipated to include sequences generated from TE insertions absent from the reference genome. Read 2 sequences generated from short library fragments could potentially contain TEnested primer sites, 3 prime TE and poly-A tail sequences either failing to map and/or capable of mapping to near-identical sequences contained in TEs present in the reference at alternative locations, including potentially on different chromosomes. Therefore, unlike standard genomic library processing, we placed no requirements for alignments to adhere to Sam specification's flags for both mates mapping, nor mapping as "proper pair", and placed no restrictions on the insert size. Unmapped reads and reads with mapping quality 0 were removed after primer and TE-like sequence requirements, to ensure that TE loci spanned by clusters of read 1 mapping reliably (minimum MAPQ> = 3) were retained for analysis.

\section{Clustering}

To identify unique TE insertions we created a catalog of filtered reads (read 1 and, potentially, read 2) covering a genomic coordinate interval (cluster), and second, used mapping signals in conjunction with genome annotations to classify predicted insertion calls.

In the first step, individual reads were clustered by genomic position for each library of each uniquely indexed sample and TE primer combination. Clusters were annotated with various attributes of the corresponding catalog of reads for use as evidence at each locus in downstream filtering (see below). Clusters were generated requiring a minimum inter-distance of $200 \mathrm{bp}$ between neighboring clusters.

Clusters with a minimum cluster size based on sequence read length (100 bp) were retained, and clusters were required to have a minimum of 2 reads derived from the TE's unique flanking sequence (read 1), having minimum mapping quality (MAPQ $\geq 3$ ).

Clusters in regions of potential noise due to read mapping artifacts were filtered. Calls in proximity to annotated reference gaps (unassembled poly-Ns; 500-bp window), regions enriched for satellite, i.e. centromeres, and sub-telomeres (defined as 1-Mb window), and located on chrY were excluded from downstream analysis (annotations obtained from UCSC Table Browser; [43]).

\section{Annotation}

In the second step, cluster loci were annotated using known TE databases [32, 34] and insertions from previously published TE assays [14, 15, 23, 25]. To achieve this, we compiled a comprehensive local database of polymorphic Alu and LINE elements called polyTEdb. The compiled polyTEdb is available for download from github (https://github.com/ekviky/TE-NGS). Clusters were intersected with annotations and labeled with TE annotation subfamily (when available); clusters mapping to within $600 \mathrm{bp}$ window of the TE $3^{\prime}$ end (when strand orientation provided) were annotated.

Calls produced by the above pipeline were classified as reference if reference genome contains insertion at the position and matches subfamily of target assay; known non-reference if evidence of an insertion of polyTE at that position in polyTEdb (because subfamily-specific information is not routinely/consistently provided). All calls lacking previous evidence of a TE insertion at that position were considered novel.

\section{Reference TE in NA12878}

Targeted loci present in hg19 were defined by identifying all matches to TE-target primers, and TE-nested primers using the parameters defined above (see Primer Design). TEs containing matches to both primers, within a maximum distance of $200 \mathrm{bp}$, and in proper order and orientation with respect to reference strand, were classified as targeted loci. Targeted loci excluding chrY, annotated reference gaps (unassembled poly-Ns; 500-bp) or centromeres/sub-telomeric regions (defined as $1-\mathrm{Mb}$ window) were retained in the Reference TE set (annotations obtained from UCSC Table Browser; [43]). Loci were further refined to exclude target loci overlapping NA12878-specific copy number variants (CNV) as 
annotated in Database Genomic Variants [49]. This resulted in 624, 2739, and 1847 reference L1HS, AluYa5/ 8 , and AluYb8/9 insertions respectively (Table 1 ).

\section{NA12878-specific TE}

Mobile element insertions ("MEI") observed in the NA12878 individual were parsed from variant vcf files obtained from Phase3 of 1000 genomes [14, 50]. $857 \mathrm{Alu}$ and 76 LINE1 remained after filtering to exclude annotated reference gaps, centromeres/sub-telomeric regions, or NA12878-specific CNV (see above definitions). Of the 857 Alu loci, 163 AluYa5/8 and 61 AluYb8/9 included subfamily designation; these insertions were used to define respective Alu false negatives in the TE-NGS call set; all 76 LINE1 calls lacked subfamily information and were used to define L1HS false negatives.

\section{Parental truth sets}

For two trios included in this study, inheritance patterns were ascertained for TEs observed in each proband as follows. The parental truth set for each trio was defined as TE insertions detected at the same location (to within a window of $100 \mathrm{bp}$ ) in both parents; $5692 \mathrm{TE}$ were present in both parents of Trio A, and 6156 for Trio B.

False positives in the proband of each trio were classified as novel TE insertions absent from both parents (i.e., apparent "de novo"). Because we observe virtually every TE in a genome with a minimum of one read (Fig. 2; see above), in defining false positives we relaxed the minimum read coverage requirement for the parental clusters and conservatively defined de novo calls in the proband as novel loci lacking evidence from even one read in one parent.

\section{Precision and recall}

To characterize the ability of TE-NGS to predict true insertions, we evaluated the sensitivity or recall, computed as $\mathrm{TP} /(\mathrm{TP}+\mathrm{FN})$, for each of the above truth sets. Precision, computed as $\mathrm{TP} /(\mathrm{TP}+\mathrm{FP})$, was used to characterize the ability of the assay to reject false insertion predictions.

\section{Validation of NA12878 calls with long read data}

Loci corresponding to potential false positive calls were inspected with two data sets generated by long-read sequencing platforms. BAM alignments provided by Genome in a Bottle (GiaB) using PacBio sequencing data were obtained from [51]. Long reads spanning TE calls were required to contain exact matches to TE-like sequences (see above).

BAM alignments of NA12878 reads generated by ONT were obtained from the Wellcome Trust Centre for Human Genetics [40]. Alignments were manually inspected by Integrated Genome Viewer (IGV) [52]. FP loci were inspected for characteristics typical of TE insertions: stretches of inserted sequence and/or clipping of long reads containing sequence absent from the reference (see Additional file 1: Figure S4).

\section{Additional file}

Additional file 1: Supplemental Material. Contains detailed procedures, all supplemental figures (Figure S1-S6), and supplemental tables (Tables S1-S4). (PDF $1569 \mathrm{~kb})$

\section{Abbreviations \\ CNV: Copy number variant; FN: False negative; FP: False positive; GiaB: Genome in a bottle; L1HS: L1 Human-specific; MEl: Mobile element insertion; NGS: Next generation sequencing; ONT: Oxford Nanopore Technology; polyTEdb: Polymorphic TE database; TE: Transposable element; TP: True positive}

\section{Acknowledgements}

We thank the patients and their families who consented to these studies. We are grateful to Andrew Wilkie, Noémi Roy, Anna Schuh, and Ed Blair for permission and use of samples. Sequencing data was generated by the High-Throughput Genomics Group at the Wellcome Centre for Human Genetics. We would like to thank HTG and the Library Prep team in particular for generous use of lab space and helpful discussions. We thank Amy Trebes, Samantha Knight, Kateryna Makova, and 2 anonymous reviewers for helpful comments on an earlier version of this manuscript. The views expressed in this manuscript are those of the authors and not necessarily the Wellcome Trust and Department of Health.

\section{Funding}

This work was supported by Wellcome Trust grant 090532/Z/09/Z.

\section{Availability of data and materials}

The datasets generated and analysed during the current study are available in the TE-NGS repository, hosted on (https://github.com/ekviky/TE-NGS).

\section{Authors' contributions}

EK, GL, PP designed the TE targeting protocol; EK, GL designed the TE detection algorithm. EK conducted the experiments, designed and wrote the code implementing the algorithm. EK, GL analyzed, interpreted the results, and wrote the manuscript. JCT provided samples. EK, PP, JCT, GL wrote, reviewed, and edited the manuscript. All authors read and approved the final manuscript.

Ethics approval and consent to participate

Genomic DNA from individual NA12878 is publicly available. For 12 the clinical samples, individual researchers had explicit research consent to undertake genetic investigation into the cause of the relevant disease. Samples provided by Anna Schuh were approved by Oxford Research Ethics Committee C (REC reference: 09/H0606/5); samples provided by Ed Blair were approved by NRES West Midlands, Coventry and Warwickshire (REC reference [13]/WM/0466). The samples provided by Andrew Wilkie were approved by London Riverside Research Ethics Committee (REC reference 09/H0706/20). Written informed consent for participation in this study was obtained from healthy donors, patients, or their parents in all cases.

\section{Consent for publication}

Not applicable

\section{Competing interests}

The authors declare that they have no competing interests.

\section{Publisher's Note}

Springer Nature remains neutral with regard to jurisdictional claims in published maps and institutional affiliations. 


\section{Author details}

${ }^{1}$ Wellcome Trust Centre for Human Genetics, Oxford, UK. ${ }^{2}$ National Institute for Health Research Comprehensive Biomedical Research Centre, Oxford, UK. ${ }^{3}$ Department of Medicine, Imperial College London, London, UK.

\section{Received: 25 September 2017 Accepted: 16 January 2018}

Published online: 01 February 2018

\section{References}

1. Consortium UK, Walter K, Min JL, Huang J, Crooks L, Memari Y, McCarthy S, Perry JR, Xu C, Futema M, et al. The UK10K project identifies rare variants in health and disease. Nature. 2015;526(7571):82-90.

2. Taylor JC, Martin HC, Lise S, Broxholme J, Cazier JB, Rimmer A, Kanapin A, Lunter G, Fiddy S, Allan C, et al. Factors influencing success of clinical genome sequencing across a broad spectrum of disorders. Nat Genet. 2015: 47(7):717-26.

3. Wright CF, Fitzgerald TW, Jones WD, Clayton S, McRae JF, van Kogelenberg M, King DA, Ambridge K, Barrett DM, Bayzetinova T, et al. Genetic diagnosis of developmental disorders in the DDD study: a scalable analysis of genome-wide research data. Lancet. 2015;385(9975):1305-14.

4. Lek M, Karczewski KJ, Minikel EV, Samocha KE, Banks E, Fennell T, O'DonnellLuria AH, Ware JS, Hill AJ, Cummings BB, et al. Analysis of protein-coding genetic variation in 60,706 humans. Nature. 2016;536(7616):285-91.

5. Lander ES, Linton LM, Birren B, Nusbaum C, Zody MC, Baldwin J, Devon K, Dewar K, Doyle M, FitzHugh W, et al. Initial sequencing and analysis of the human genome. Nature. 2001:409(6822):860-921.

6. de Koning AP, Gu W, Castoe TA, Batzer MA, Pollock DD. Repetitive elements may comprise over two-thirds of the human genome. PLoS Genet. 2011; 7(12):e1002384.

7. Sheen FM, Sherry ST, Risch GM, Robichaux M, Nasidze I, Stoneking M, Batzer MA, Swergold GD. Reading between the LINEs: human genomic variation induced by LINE-1 retrotransposition. Genome Res. 2000;10(10):1496-508.

8. Boissinot S, Chevret P, Furano AV. L1 (LINE-1) retrotransposon evolution and amplification in recent human history. Mol Biol Evol. 2000;17(6):915-28.

9. Brouha B, Schustak J, Badge RM, Lutz-Prigge S, Farley AH, Moran JV Kazazian HH Jr. Hot L1s account for the bulk of retrotransposition in the human population. Proc Natl Acad Sci U S A. 2003;100(9):5280-5.

10. Jurka J. Sequence patterns indicate an enzymatic involvement in integration of mammalian retroposons. Proc Natl Acad Sci U S A. 1997:94(5):1872-7.

11. Cost GJ, Feng Q, Jacquier A, Boeke JD. Human L1 element target-primed reverse transcription in vitro. EMBO J. 2002;21(21):5899-910.

12. Cordaux R, Hedges DJ, Herke SW, Batzer MA. Estimating the retrotransposition rate of human Alu elements. Gene. 2006;373:134-7.

13. Kazazian $\mathrm{HH} \mathrm{Jr}$. An estimated frequency of endogenous insertional mutations in humans. Nat Genet. 1999;22(2):130.

14. Sudmant PH, Rausch T, Gardner EJ, Handsaker RE, Abyzov A, Huddleston J, Zhang Y, Ye K, Jun G, Hsi-Yang Fritz M, et al. An integrated map of structural variation in 2,504 human genomes. Nature. 2015;526(7571):75-81.

15. Stewart C, Kural D, Stromberg MP, Walker JA, Konkel MK, Stutz AM, Urban AE, Grubert F, Lam HY, Lee WP, et al. A comprehensive map of mobile element insertion polymorphisms in humans. PLoS Genet. 2011; 7(8):e1002236

16. Kazazian HH Jr. Mobile elements: drivers of genome evolution. Science. 2004;303(5664):1626-32.

17. Callinan PA, Batzer MA. Retrotransposable elements and human disease. Genome Dyn. 2006;1:104-15.

18. Hancks DC, Kazazian HH Jr. Roles for retrotransposon insertions in human disease. Mob DNA. 2016;7:9.

19. Badge RM, Alisch RS, Moran JV. ATLAS: a system to selectively identify human-specific L1 insertions. Am J Hum Genet. 2003;72(4):823-38.

20. Wheelan SJ, Scheifele LZ, Martinez-Murillo F, Irizarry RA, Boeke JD. Transposon insertion site profiling chip (TIP-chip). Proc Natl Acad Sci U S A. 2006;103(47):17632-7.

21. Iskow RC, McCabe MT, Mills RE, Torene S, Pittard WS, Neuwald AF, Van Meir EG, Vertino PM, Devine SE. Natural mutagenesis of human genomes by endogenous retrotransposons. Cell. 2010;141(7):1253-61.

22. Ewing $A D$, Kazazian $H H$ Jr. High-throughput sequencing reveals extensive variation in human-specific L1 content in individual human genomes. Genome Res. 2010;20(9):1262-70.
23. Ewing $A D$, Kazazian $H H$ Jr. Whole-genome resequencing allows detection of many rare LINE-1 insertion alleles in humans. Genome Res. 2011;21(6):985-90

24. Witherspoon DJ, Xing J, Zhang Y, Watkins WS, Batzer MA, Jorde LB. Mobile element scanning (ME-scan) by targeted high-throughput sequencing. BMC Genomics. 2010;11:410.

25. Witherspoon DJ, Zhang Y, Xing J, Watkins WS, Ha H, Batzer MA, Jorde LB. Mobile element scanning (ME-scan) identifies thousands of novel Alu insertions in diverse human populations. Genome Res. 2013;23(7): 1170-81

26. Tang Z, Steranka JP, Ma S, Grivainis M, Rodic N, Huang CR, Shih IM, Wang TL, Boeke JD, Fenyo D, et al. Human transposon insertion profiling: analysis, visualization and identification of somatic LINE-1 insertions in ovarian cancer. Proc Natl Acad Sci U S A. 2017;114(5): E733-40.

27. Shukla R, Upton KR, Munoz-Lopez M, Gerhardt DJ, Fisher ME, Nguyen T, Brennan PM, Baillie JK, Collino A, Ghisletti S, et al. Endogenous retrotransposition activates oncogenic pathways in hepatocellular carcinoma. Cell. 2013;153(1):101-11.

28. Streva VA, Jordan VE, Linker S, Hedges DJ, Batzer MA, Deininger PL. Sequencing, identification and mapping of primed L1 elements (SIMPLE) reveals significant variation in full length $\mathrm{L} 1$ elements between individuals. BMC Genomics. 2015;16:220.

29. Xing J, Witherspoon DJ, Jorde LB. Mobile element biology: new possibilities with high-throughput sequencing. Trends Genet. 2013;29(5):280-9.

30. Stenger JE, Lobachev KS, Gordenin D, Darden TA, Jurka J, Resnick MA. Biased distribution of inverted and direct Alus in the human genome: implications for insertion, exclusion, and genome stability. Genome Res. 2001;11(1):12-27.

31. Kvikstad EM, Makova KD. The ( $r$ ) evolution of SINE versus LINE distributions in primate genomes: sex chromosomes are important. Genome Res. 2010; 20(5):600-13.

32. Smit AF, Hubley R, Green P. RepeatMasker Open-4.0. 2013-2015.

33. Bao W, Kojima KK, Kohany O. Repbase update, a database of repetitive elements in eukaryotic genomes. Mob DNA. 2015;6:11.

34. Wang J, Song L, Grover D, Azrak S, Batzer MA, Liang P. dbRIP: a highly integrated database of retrotransposon insertion polymorphisms in humans. Hum Mutat. 2006:27(4):323-9.

35. Boissinot S, Entezam A, Furano AV. Selection against deleterious LINE-1containing loci in the human lineage. Mol Biol Evol. 2001;18(6):926-35.

36. Rishishwar L, Marino-Ramirez L, Jordan IK. Benchmarking computational tools for polymorphic transposable element detection. Brief Bioinform. 2016:18(6):908-18

37. Rhoads A, Au KF. PacBio sequencing and its applications. Genomics Proteomics Bioinformatics. 2015;13(5):278-89.

38. Boissinot S, Entezam A, Young L, Munson PJ, Furano AV. The insertional history of an active family of $L 1$ retrotransposons in humans. Genome Res. 2004;14(7):1221-31.

39. Eid J, Fehr A, Gray J, Luong K, Lyle J, Otto G, Peluso P, Rank D, Baybayan P, Bettman $B$, et al. Real-time DNA sequencing from single polymerase molecules. Science. 2009;323(5910):133-8.

40. WTCHG [www.well.ox.ac.uk/ogc/nanopore-data]

41. Ewing AD. Transposable element detection from whole genome sequence data. Mob DNA. 2015;6:24

42. Untergasser A, Cutcutache I, Koressaar T, Ye J, Faircloth BC, Remm M, Rozen SG. Primer3-new capabilities and interfaces. Nucleic Acids Res. 2012;40(15):e115.

43. Karolchik D, Hinrichs AS, Furey TS, Roskin KM, Sugnet CW, Haussler D, Kent WJ. The UCSC table browser data retrieval tool. Nucleic Acids Res. 2004; 32(Database issue):D493-6.

44. Li H, Durbin R. Fast and accurate short read alignment with burrowswheeler transform. Bioinformatics. 2009:25(14):1754-60.

45. Lunter G, Goodson M. Stampy: a statistical algorithm for sensitive and fast mapping of Illumina sequence reads. Genome Res. 2011;21(6):936-9.

46. Li H, Handsaker B, Wysoker A, Fennell T, Ruan J, Homer N, Marth G, Abecasis G, Durbin R, Genome Project Data Processing S. The sequence alignment/ map format and SAMtools. Bioinformatics. 2009:25(16):2078-9.

47. Picard [http://broadinstitute.github.io/picard/].

48. Edgar RC. MUSCLE: multiple sequence alignment with high accuracy and high throughput. Nucleic Acids Res. 2004;32(5):1792-7. 
49. MacDonald JR, Ziman R, Yuen RK, Feuk L, Scherer SW. The database of genomic variants: a curated collection of structural variation in the human genome. Nucleic Acids Res. 2014;42(Database issue):D986-92.

50. Genomes Project C, Auton A, Brooks LD, Durbin RM, Garrison EP, Kang HM, Korbel JO, Marchini JL, McCarthy S, McVean GA, et al. A global reference for human genetic variation. Nature. 2015;526(7571):68-74.

51. GiaB [(ftp://ftp-trace.ncbi.nlm.nih.gov/giab/ftp/data/NA12878/NA12878_ PacBio_MtSinai/)].

52. Robinson JT, Thorvaldsdottir H, Winckler W, Guttman M, Lander ES, Getz G, Mesirov JP. Integrative genomics viewer. Nat Biotechnol. 2011;29(1): 24-6

Submit your next manuscript to BioMed Central and we will help you at every step:

- We accept pre-submission inquiries

- Our selector tool helps you to find the most relevant journal

- We provide round the clock customer support

- Convenient online submission

- Thorough peer review

- Inclusion in PubMed and all major indexing services

- Maximum visibility for your research

Submit your manuscript at www.biomedcentral.com/submit 